\title{
IMPROVEMENT IN THE GEOFENCING SERVICE INTERFACE USING INDOOR POSITIONING SYSTEMS AND MOBILE SENSORS
}

\author{
Masafumi Nakagawa \\ Dept. of Civil Engineering, Shibaura Institute of Technology, 3-7-5, Toyosu, Koto-ku, Tokyo 135-8548 Japan - \\ mnaka@shibaura-it.ac.jp
}

Commission IV / WG 7

KEY WORDS: Location-based services, Geofencing service, Indoor positioning, Indoor message system, Accelerometer, Mobile user, Notifications

\begin{abstract}
:
The current state of location-based services provides spatial information delivery for mobile users based on position data taken from GPS sensors. However, sometimes the spatial information delivery service includes unwanted information. In particular, push-based or passive information delivery has a high probability that users receive unwanted information. We propose a new spatial information delivery to improve the integrity of spatial information delivery. We conducted an experiment using an Indoor Messaging System and an accelerometer, and concluded that our methodology can detect user behavior without accessing personal information and reduce the amount of spam information.
\end{abstract}

\section{INTRODUCTION}

Recently, Global Positioning System (GPS) receivers have been mounted on smartphones, cellphones, and tablet PCs. In an outdoor environment, position data can be acquired with more than four visible GPS satellites Moreover, acceleration and magnetic direction sensors are mounted on recent smartphones and tablet PCs. In the current state, assisted GPS is provided to improve accuracy and availability in positioning. The indoor messaging system (IMES) (Manandhar et al. 2008), Wi-Fi (Liu et al. 2012), radio frequency identification (RFID), and Bluetooth have also been developed for use as indoor positioning and navigation services. These indoor systems with Global Navigation Satellite Systems including GPS can provide indoor-outdoor seamless positioning and navigation services (Petri et al.2004).

Mobile hardware development has improved the availability of positioning services, which requires quality improvement of the positioning services themselves. Although positioning accuracy is one of the important issues in location-based services, many other significant issues, such as availability, integrity, and reliability must be considered in quality improvement of positioning services, including information distribution.

There are two types of information distribution in internet services. One is pull-based distribution as a passive download. In pull-based distribution, the user clicks a button, such as an icon or link to download a file or service, and they can choose to download a variety of spatial content through push-based information distribution. The pull-based distribution is not limited to internet content. For example, augmented reality applications using a camera mounted on a mobile interface have been proposed for use in shopping malls and museums for browsing spatial information (Debenham et al. 2011). The other type is push-based distribution as an active download (Laasonen et al. 2004), in which the service provider compels the user to download a file or service from a server.

Both types of distribution are performed using user location data in location-based services. Generally, push-based distribution is more convenient than pull-based distribution because the user can enjoy automated delivery of spatial contents. However, push-based distribution has a higher probability of including unexpected information for users.

The unexpected information includes data that are unnecessary, of no interest, deceptive, or erroneous. Sometimes, attractive information can be found in the unexpected information. However, the user usually receives unwanted information, such as fishing mails and advertisements of no interest. Although conventional security software can prevent users from receiving risky mails, the software has difficulties in rejecting advertisements of no interest to the user. Frequent reception of unwanted information would drastically worsen the usability of mobile interfaces compared with a desktop PC. Moreover, the conventional software also has difficulties in controlling the timing for receiving notifications. These issues would reduce the usability of positioning services.

We propose a geofencing application as a push-based distribution paradigm in location-based services. The geofencing application uses user location data and a predefined set of boundaries on a map (Figure 1). First, a geofence is generated on a map at a radius around a store or a point location. The geofence is a virtual parameter for a real-world geographic area. Next, the geofencing application provides various services using the geofence. When a user with a location-aware device enters or exits the geofence, the mobile device receives notification such as location-based assistance or alerts via email or SMS, e.g., location-based burglar alarm, location-based schedule assistance, or cashless purchase applications. Indeed, for these applications, the availability of positioning using location-aware mobile devices is required.

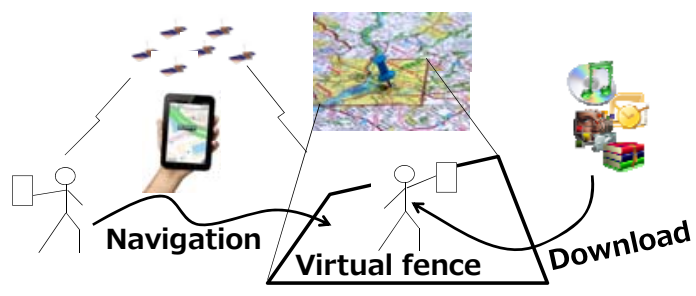

Figure 1. Geofencing service 
Location-based services, such as geofencing applications, require the achievement of higher usability and comfort with the filtering of unexpected information distribution. Moreover, to achieve higher safety in traffic assistance and autonomous robot services, location-based services should also reduce the distribution of unexpected information.

Based on these points, we mainly focus on reliability improvement of spatial information distribution that is suitable for indoor-based services. We also focus on the selection of information required by the users and the reduction of invalid information distribution.

\section{METHODOLOGY}

In conventional research, there are two approaches for achieving distribution of valid spatial contents to users. One approach is to select information for distribution using user position and stay time. The other is to classify between a walk and stop using a peak or power spectrum of data taken from an acceleration sensor. Both approaches use personal parameters such as a schedule, personal preferences, daily pattern, walk pattern, gender, and age to improve the accuracy of information selection. Many sensitive parameters need to be adjusted for individual users. On the other hand, users should be protected against leakage of personal data. These limitations make such methods unsuitable for public and anonymous services, such as museum applications and asset management.

Thus, we propose an approach for user behavior estimation using location and acceleration sensors without individual parameters to improve spatial information distribution. The processing flow is described in Figure 2.

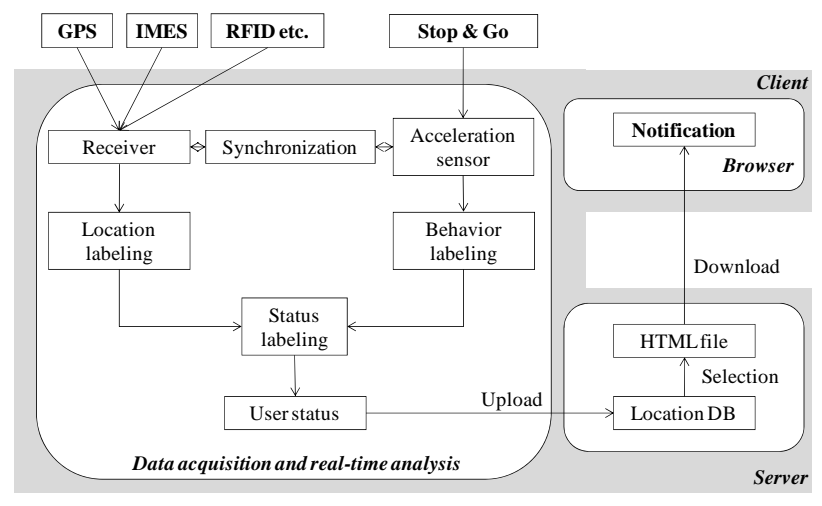

Figure 2. The processing flow with five components

\subsection{Position and acceleration data acquisition}

We used a location and acceleration sensors simultaneously. Spatial coordinate values such as longitude and latitude were acquired using GPS, RFID, Wi-Fi, and IMES receivers. Moreover, to estimate user behavior, acceleration data were acquired using an acceleration sensor mounted on a mobile device.

\subsection{Location and behavior data labeling}

Location estimation based on wireless communication depends on the stability of signals from many transmitters. In indoor environments, even if a user stops receiving signals to detect location, stable location estimation is difficult for multipath and interference of signals for positioning in the wireless communication. Thus, discontinuous fragments are generated after signal labeling using static threshold values of signal intensity. The discontinuous fragments are too unstable to be used as location data for mobile devices. Therefore, the discontinuous fragments are clustered to generate groups to be used as more stable location data (Figure 3). The acquired behavior data are also labeled and clustered to generate behavior indices consisting of the user's walk-and-stop location.

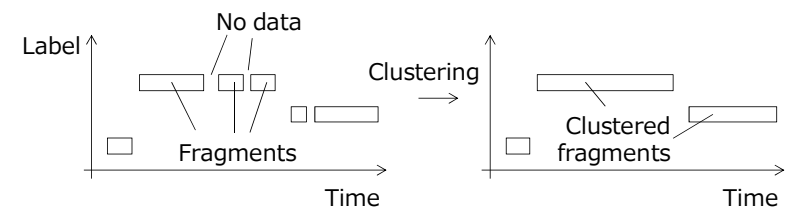

Figure 3. Labeling and clustering

\subsection{User status estimation for the best notifications}

The local situation of the user, such as walking or stopped in front of something, is defined as the user status index in this study. The user status index was estimated using location and behavior labels. This status was used to select the information required for the most useful notifications.

First, the user status index is uploaded from a user or client to a server. Next, the user status index is matched with a file such as a map, advertisement, and coupon on the server, after which the selected file is sent or distributed to the user or client to be browsed as notifications. The timing of distribution can be controlled according to the distributed file types.

\section{EXPERIMENT}

We used GPS and IMES receivers (Tokyo University of Marine Science and Technology, and JAXA) as location sensors. In addition, we used MTi (Xsens) as a three-axis acceleration sensor. IMES is one of the indoor positioning systems developed by JAXA, and it uses a signal compatible with GPS. Moreover, IMES transmitters are identified using an assigned pseudo random number (PRN). Thus, the IMES can share hardware such as an antenna and receiver with GPS. We can use RFID, Wi-Fi, and Bluetooth as indoor positioning systems in our approach. However, we focused on the advantage of seamless use in a mobile interface. Therefore, we selected IMES as the indoor positioning system in our experiment.

First, IMES transmitters and antennae were arranged in an indoor environment, as shown in Figure 4.

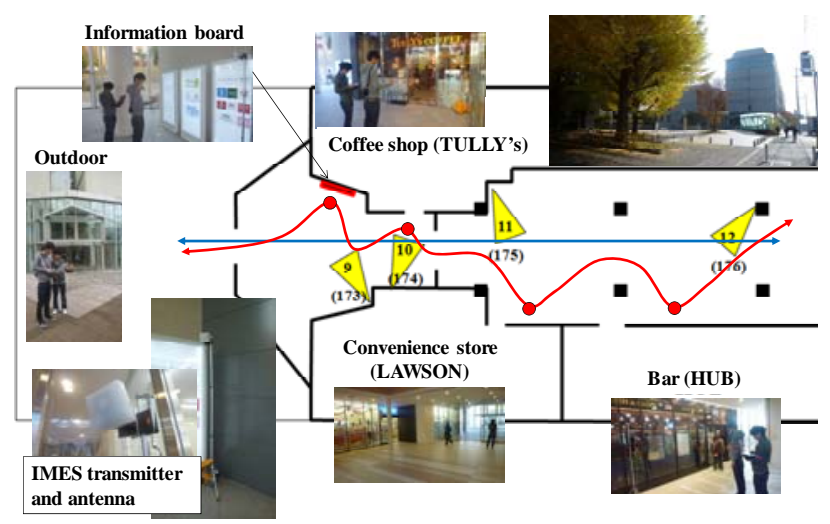

Figure 4. Data acquisition route and IMES antennae arrangement 
Next, we defined two rules. The first rule was to avoid sending spatial information to walking users. The second rule was to send spatial information to users stopping at a geofence. Based on these rules, we tested two cases consisting of "pass by each geofence" and "stop at the geofence for 10 s." Then, we conducted experiments to verify the possibility of dynamic information distribution such as "the server cancels sending spatial information for walkers" and "the server sends spatial information for uses stopping at the geofence."

We selected a commercial floor in the campus as our study area. As shown in Figure 4, we prepared five checkpoints as geofences, namely "outdoor," "information board," "coffee shop (TULLY's)," "convenience store (LAWSON)," and "bar (HUB)." The IMES transmitters at the checkpoints of information board, coffee shop, convenience store, and bar were assigned PRNs between 173 and 176, respectively.

\subsection{Location and behavior data acquisition}

We combined the IMES receiver and the three-axis acceleration sensor on a wooden board a little larger than a smartphone, as shown in Figure 5. The IMES receiver acquired short identification data from the IMES transmitters at $1 \mathrm{~Hz}$. The acceleration sensor acquired acceleration data at $100 \mathrm{~Hz}$. The receiver and acceleration sensors were connected to a notebook PC via USB cables and their data were acquired simultaneously. A tester walked in the indoor and outdoor environments through each geofence carrying these combined sensors.
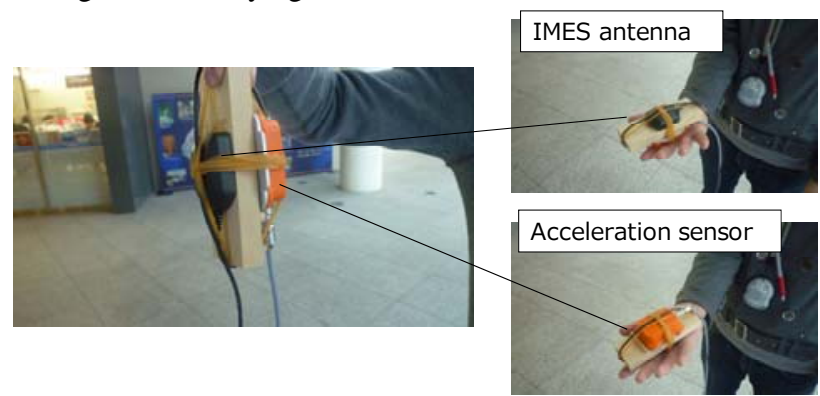

Figure 5. Combination of IMES receiver and acceleration sensors

In addition, a local server was prepared in the notebook PC to achieve selection and distribution of spatial files based on location. The IMES receiver was assigned eight channels for GPS signals and four channels for IMES signals. In this experiment, positioning calculation was omitted in IMES positioning. Instead, the PRN identifiers of the IMES transmitters were assigned as floor identifiers describing geofences and checkpoints.

\subsection{Location and behavior data labeling}

The PRN identifier of the strongest IMES signal was used as position data. The PRN identifier was translated into the checkpoint identifier number, such as the information board. However, IMES signals were too unstable to identify the strongest signal in real time. Therefore, the IMES signals received from transmitters over a few seconds were used to calculate temporal average values in each channel. After that, the strongest and continuous signal was detected from the averaged results. On the other hand, when there were no signals or low intensity values from the IMES transmitters, we assumed the user was outside the geofences.

A waveform in the acceleration data can represent user's movements. However, our approach requires real-time classification between a walk, a stop, or noise. In our experiment, temporal averages of acceleration data in one second were calculated to recognize user behavior such as walk and stop with noise filtering. When the average was higher than a certain threshold, we assumed that the user's status was "walk." The threshold was set as $0.2 \mathrm{~m} / \mathrm{s}^{2}$ from our preliminary examination.

\subsection{User status from location and behavior data}

A combination of a stop status and the user's position at the geofence was translated into a user status that "the user has stopped at geofence." Although the timing of the distribution of spatial content is a flexible parameter, the time to start a distribution of file was set as $3.0 \mathrm{~s}$ for all spatial content after the user status recognition in our experiment.

\subsection{Information selection for notifications}

Suitable distribution information for the user was selected from prepared web files as shown in Figure 6. Next, the selected web file was browsed on the mobile interface as notification. In a similar way to location-based applications, the user can browse only web files appropriate to their position, as reported by the hidden status of IMES signals and acceleration data.

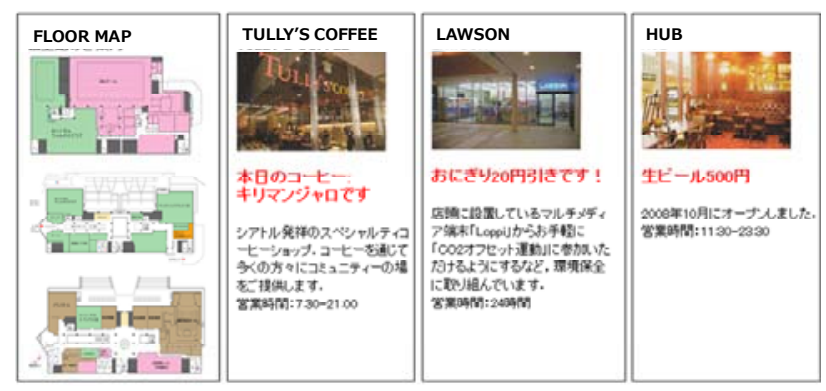

Figure 6. Prepared web files

Synchronized IMES signals and acceleration data are shown in Figure 7. The horizontal axis indicates the data acquisition time. The top image in Figure 7 shows the signal intensity values of GPS and IMES. The vertical axis in the top image in Figure 7 indicates 12 channels in the receiver. We confirmed that IMES signals were received from channel 9 to 12 successfully. The bottom image in Figure 7 shows acceleration data using the $\mathrm{MTi}$. The vertical axis in this image indicates relative acceleration values rectified with the gravity acceleration value.

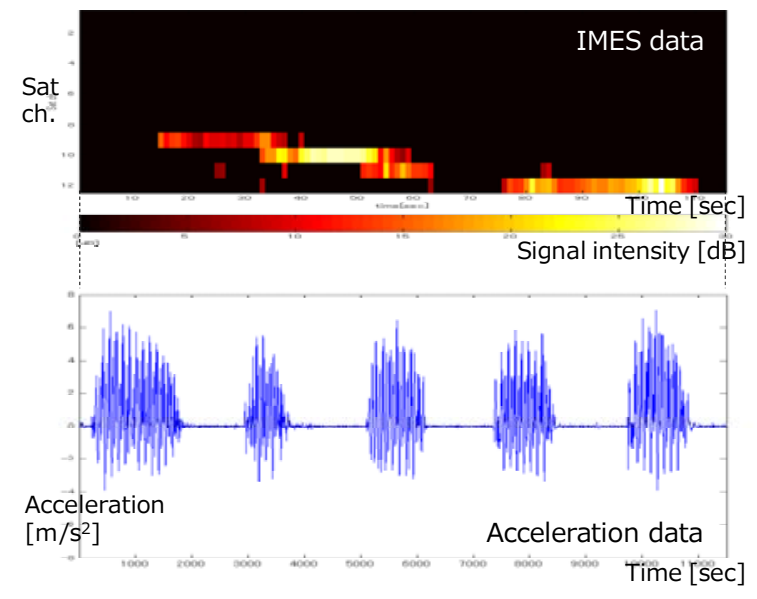

Figure 7. Synchronized IMES and acceleration sensor data 
A location was detected using the PRN identifier number of the strongest signal from several IMES transmitters to define proximity to a checkpoint. Then, the estimated location was translated into a checkpoint identifier number. Figure 8 shows a location detection result based on a translation from PRN to the checkpoint identifier number. When a user stopped at a geofence such as the information board, the coffee shop, the convenience store, or the bar, the location was successfully estimated at each point. When there were no signals or low intensity values from IMES transmitters, the user was also recognized as being out of the geofences.

In addition, Figure 8 shows that the user status such as walk and stop was recognized in selecting a web file for distribution to the user. First, a combination of stop status and location data generated a context as "a user has stopped at the geofence". Then, based on this context, suitable web files for the user were presented on a browser after time $T_{1}, T_{2}, T_{3}$, and $T_{4}[s]$ as pushbased information distribution.

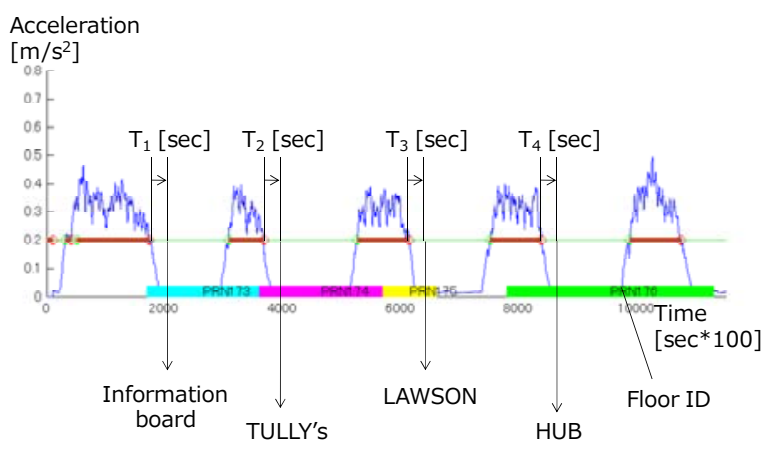

Figure 8. Result of status translation and spatial data distribution

\section{DISCUSSION}

The following three benefits are expected from our approach. First, the user can automatically receive only information of interests, based on dynamic information selection corresponding with their status. For example, when the user stops to look at an advertisement in front of a store, we would receive a recommendation or coupon from the store. On the other hand, when the user passes by the store, the recommendation would be rejected as ineffective information. Moreover, the store can send the user suitable advertisements according to their status. As a result, unwanted information can be reduced for individual users.

Second, we can detect a user's status without personal parameters. Generally, location-based services require private information such as schedules, preferences, genders, and ages to improve accuracy of user status in personal use. By contrast, we can achieve a precise information distribution using location and acceleration data.

Third, we can estimate user status using sensors mounted on mobile interfaces. Although we require the preparation of spatial infrastructure such as transmitters for indoor positioning, our approach can improve the performance of user status estimation using low-cost sensors mounted on mobile interfaces without precise maps.

Our approach requires users simultaneous acquisition of location and acceleration data. First, we confirmed that IMES signals were received at each checkpoint, indicating that sequential user positions were estimated. Moreover, Figure 5 shows that three-axis acceleration data can classify user behavior between walk and stop clearly. Although a more detailed behavior estimate would require personal parameter adjustments, we confirmed that our approach can estimate the user status without personal parameters in real time.

We can summarize the findings in our experiments as follows. First, when a user comes and goes in the same area frequently, a positional log such as the PRN of IMES is recorded for a while to invalid information distribution. Second, when a serviced area experiences interference and multipaths of IMES signals, the strongest and continuous IMES signal at the time should be selected as a valid signal for position estimation. A delay parameter was set as $3.0 \mathrm{~s}$ for suitable signal estimation in our experiment. We have confirmed that location estimation using momentary IMES signal intensity was unstable in indoor environments because of the interference and multipath.

\section{SUMMARY}

We have focused on an improvement in spatial information distribution for mobile interfaces in indoor environments and proposed an approach to estimate user status using location and acceleration data taken from mobile interfaces, without personal data, to achieve precise reporting of spatial information. Moreover, we confirmed that our approach can achieve dynamic information selection based on a combination between the IMES and acceleration sensors in an indoor experiment. We have two issues for our future work. The first issue is a more suitable arrangement of IMES transmitters and antennae to avoid IMES signal interference and multipath. The second issue is to automate the calibration of the IMES signal power in the arrangement of IMES transmitters for indoor seamless positioning using various indoor positioning systems.

\section{REFERENCES}

Manandhar, D., Kawaguchi, S., Uchida, M., Ishii, M., Torimoto, H., 2008, IMES for Mobile Users Social Implementation and Experiments based on Existing Cellular Phones for Seamless Positioning, Proceedings of International Symposium 2008 on GPS-GNSS.

Liu, H., Gan, Y., Yang, J., Sidhom, S., Wang, Y., Chen, Y., Ye, F., 2012, Push the limit of WiFi based localization for smartphones, Mobicom '12 Proceedings of the 18th annual international conference on Mobile computing and networking, pp. 305-316.

Petri, K., Petri, M., Teemu, R., Henry, T., Kimmo, V.Hannes, W. 2004. Topics in probabilistic location estimation in wireless networks. In: Personal, Indoor and Mobile Radio Communications, 2004. PIMRC 2004. 15th IEEE International Symposium, Vol.2, pp. 1052-1056.

Debenham, P., Thomas, G., Trout, J., 2011, Evolutionary Augmented Reality at the Natural History Museum, $B B C R \& D$ White Paper (Nov. 2011), WHP212, British Broadcasting Corporation, pp.1-12.

Laasonen, K., Raento, M., Toivonen, H., 2004, Adaptive ondevice location recognition, In: Proceedings of the Second International Conference on Pervasive Computing, Vol. 3001 of Lecture Notes in Computer Science, Springer-Verlag, pp. 287304. 